\title{
Inter-Company Supply Chains Integration via Mobile Agents
}

\author{
D. Brugali, G. Menga, S. Galarraga \\ Politecnico di Torino \\ C.so Duca degli Abruzzi, 24 \\ 10129 Torino - Italy \\ tel. +39011 564 7012, fax. (7099) \\ \{brugali,menga, stefanog\}@polito.it
}

\begin{abstract}
In this paper we show how we have applied, in a current project, mobile agent technology to an industrial process in the field of textile manufacturing. The project involves several companies, each of which is a Europe-wide representative of one of the key phases of the textile production process (e.g. spinning, weaving, dyeing, finishing and ready made clothing). The main contribution of this paper is the description of the agent-oriented application framework which integrates their business enterprise information systems, to create a supply and distribution chain in the volatile (seasonal) environment of top fashion men's and women's wool garment manufacturing.
\end{abstract}

\section{Keywords}

Virtual Factories, Mobile Agents, textile manufacturing process

\section{INTRODUCTION}

As pointed out in (Deis, 1996), there has been a series of global, massive changes in recent years in the business environment of Computer Integrated Manufacturing (CIM). Production is now characterized by smaller lots with wider variation; the market changes more rapid and has shorter lead times; companies rely on extensive outsourcing instead of internal production.

These changes have led to an increasing need to integrate process chains beyond company boundaries to customers and suppliers, in order to create network-wide integrated distributed Virtual Factories (VFs) (Raulefs, 1994, Nau, 1997). 
In (Bell, 1996) a VF is defined as "a community of dozens of factories each focused on what it does best, all linked by an electronic network that would enable them to operate as one -- flexibly and inexpensively regardless of their location".

A Virtual Factory is a specific example of a distributed system which supports highly specialized, concurrent and distributed task-planning and decision making, integrating enterprise-wide business functions with Flexible Manufacturing Systems (FMS).

In order to ensure coherence in problem-solving and decision making and to guarantee that end-users and application designers use a VF effectively, a technology which allows geographically dispersed activities to be flexibly combined and coordinated is necessary.

The goal of this paper is to show how we have applied Agent Technology (for a survey see (Riecken, 1994)) and Code Mobility (for a survey see (Ghezzi and Vigna, 1997) in the development of a communication and coordination infrastructure between distributed information systems. The infrastructure integrates the production planning and marketing activities of several European textile manufacturing factories involved in a current ESPRIT project.

The main contribution of this paper is the description of the agent-based application framework, which has been developed during the project.

The paper is organized as follows. Section 2 analyses the requirements and the characteristics of the communication and coordination infrastructure that we have developed in order to integrate distributed supply chains. Section 3 describes the design of the application framework which implements the integration infrastructure. Section 4 documents some related work. Finally, Section 5 draws some conclusions.

\section{PRODUCTION AND SUPPLY ELECTRONIC CHAIN}

The project under consideration will create a supply and distribution chain in the volatile (seasonal) environment of high fashion men's/women's wool garment manufacturing. The cycle of textile processes, from fiber to garment is made up of a long chain of operations grouped in a few important stages: spinning, weaving, dyeing, finishing and ready made or made-to-measure clothing.

Large fully integrated companies performing the full cycle are very rare. The textile industry is typically conservative, and liaisons amongst different firms are generally based on traditional schemes and methods; therefore quick response and continuous swift adaptation of production planning in real time are little known and seldom applied.

This situation constitutes a serious handicap, especially in the worsted and woollen industry, whose high priced goods, with a strong percentage of fancy colours and patterns are manufactured. The market is characterized by rapid changes of fashion, style and look. This in turn requires deliveries within a period of weeks. This factor compels the operators in the different branches in the cycle to look for ways of establishing closer integration and creates the need for speed in communication.

The market requirement is therefore for an interconnecting network where product data coupled with a strongly integrated scheduling of the production 
between retailers, the garment producer and its suppliers, commission transformers and customers, reduces the time between securing an order and delivering the product to a minimum.

Our contribution to the project has provided the architectural infrastructure that allows the different local factories to automate the process of cooperation and it consists of:

- A distributed Product Data Management (PDM) system accessible to all of the partners. Upstream (wool-spinning) the chain will respond mostly to the exchange of technical data with the objective of facilitating commercial transactions and identifying the product better. Product quality and technical aspects, quality and environmental (eco labelling) data will by-pass the manufacturer (in other words it will no longer be under the sole control of the manufacturer) and will flow to weavers and spinners directly. The PDM system will allow garment product data right to be tracked up to the wool data.

- A distributed planning and scheduling system. In the center of the chain (spinning-weaving-manufacturing) the emphasis will be mostly on ordermaterial management aspects. This part of the chain will stress and demonstrate the viability of distributed planning and it will be mostly responsible for the quick response capabilities expected from the system.

- An interactive access system to the supply chain through the Web. Downstream the chain will be characterized by the interaction between retailers and manufacturers. The framework raises the inter-factory relationship from the current file transfer level, established by private networks with added value services, to the level of direct interaction between partners offering each partner the advantage of operating directly on the other's facilities.

The framework has been designed in such a way that it meets the following specific requirements of the distributed information systems for the industry:

- Heterogeneity. A VF integrates legacy systems which are heterogeneous and not designed to be interactive. They are usually implemented using different technology, different languages and for different operating platforms. In particular, the local factories in the supply chain have heterogeneous resource planning systems.

- Scalability. A VF should be able to integrate new applications into the distributed system without affecting the other components of the system.

- Reconfigurability. The interactions between the local factories of the supplychain should be flexible and easy to accommodate to new requirements. In many cases it is not possible to interrupt the production process, shut down the entire system and reconfigure it off-line. In particular, the enterprise resource planning system should be able to react to the dynamics of the market (changes due to strikes, modifications in exchange rates etc.).

- Easy accessibility. The distributed and heterogeneous nature of the system should be hidden to the user who must be able to access its services in a homogeneous way. 
- Performance. Communication between local factories may induce a high network traffic. It is necessary to speed up the exchange of information among the enterprises of the supply chain.

- Security. The factories belonging to the supply chain interacts through a common medium, i.e. Internet. The communication infrastructure should protect them against unauthorized attempts to access information or interfere with their operations.

\section{THE FRAMEWORK}

The supply-chain infrastructure has been designed as an organization of cooperating agents, i.e. integrated software units of design that can be flexibly composed with other similar units to build complex systems. From a structural point of view, the agents of our framework closely resemble objects as defined by the Object-Oriented Technology:

- Like active objects (Minoura et al., 1993), agents encapsulate and manage one or more independent threads of control. They can act on behalf of another agent (human or software) providing services on demand, they can react to external or internal events (such as a clock), or they can perform background activities such as monitoring their own activity or the external environment.

- Agents have an identity which distinguishes one agent from the other agents in the same system and it is expressed in terms of a name, an address, and a description of the services it provides (Brugali and Sycara, 1998).

- Agents are static, i.e. they can not autonomously change their location. However, they can be added or removed from the system at run-time, and their identity can be communicated to other agents.

- Agents have reasoning capabilities which determine their behavior; the agent's behavior is specified in a declarative way (by rules, constraints, and goals) and may change dynamically at run-time.

Interactions among agents are established dynamically according to the dependencies among their functionality. The same capability may be provided by different agents and the same agent may provide several capabilities.

Agents can cooperate since they share the same communication language and a common vocabulary, which contains words appropriate to common application areas and whose meaning is defined in a shared ontology (Genesereth, 1994).

In our framework, agents communicate using code mobility as well: the content of a message is an entire program with a complexity which can range from simple procedures to objects with state and behavior (mobile agents). Code mobility is achieved using the IBM Aglets framework (Lange and Oshima, 1998) based on the Java language. Mobile agents have a behavior, a state and a location. They are able to migrate from one static agent to another one. 


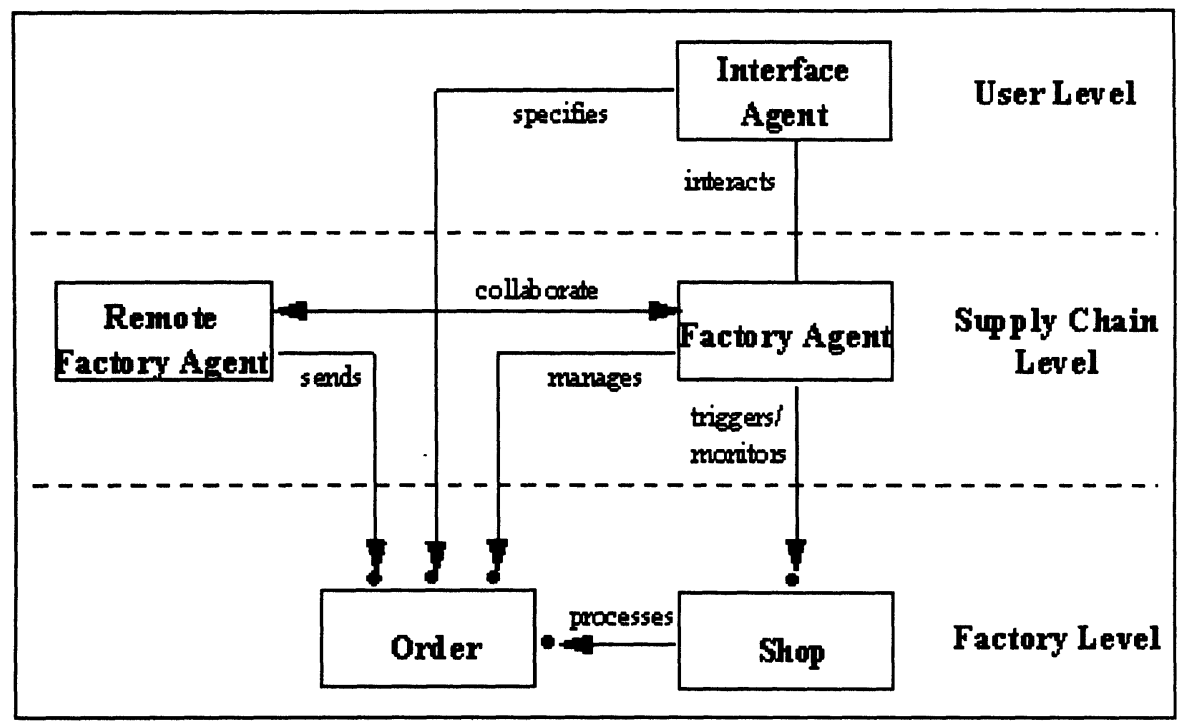

Figure 1. The Analysis of the integration infrastructure.

Our agent-based framework consists in a set of static and mobile agents organized in three functional levels (see Figure 1):

- Supply Chain Level. A set of static agents (Factory Agents) operate the physical factories which are part of the supply chain. They manage product, business, and information models and integrate the factory's business functions (order management) with its Enterprise Resource Planning (ERP) system. They reside on computers tightly coupled to the factories they operate and provide the execution environment for the mobile agents which migrate from other Factory Agents.

- Factory Level. A physical factory is usually organized in a hierarchy of control modules with different responsibilities allocated to each level (Aarsten et al. 1996). At the highest level the Facility represents one complete production system and performs long-term, strategic planning. It communicates orders to a shop floor module which schedules production lots (a lot is an administrative entity indicating a group of identical pieces to be manufactured together) and coordinates the factory's FMS.

- User Level. The users access the services provided by the VF through a Web browser, which hosts mobile agents called Interface Agents. They help and guide the users in retrieving and using services and information through the supply chain. They interact with the Factory Agents in order to post the customer's orders.

The separation into three functional layers increases the flexibility of the multiagent organization. New users can access the distributed system through new Interface Agents, new collaboration protocols can be shared by the Factory Agents, and new physical factories can become part of the supply chain. 


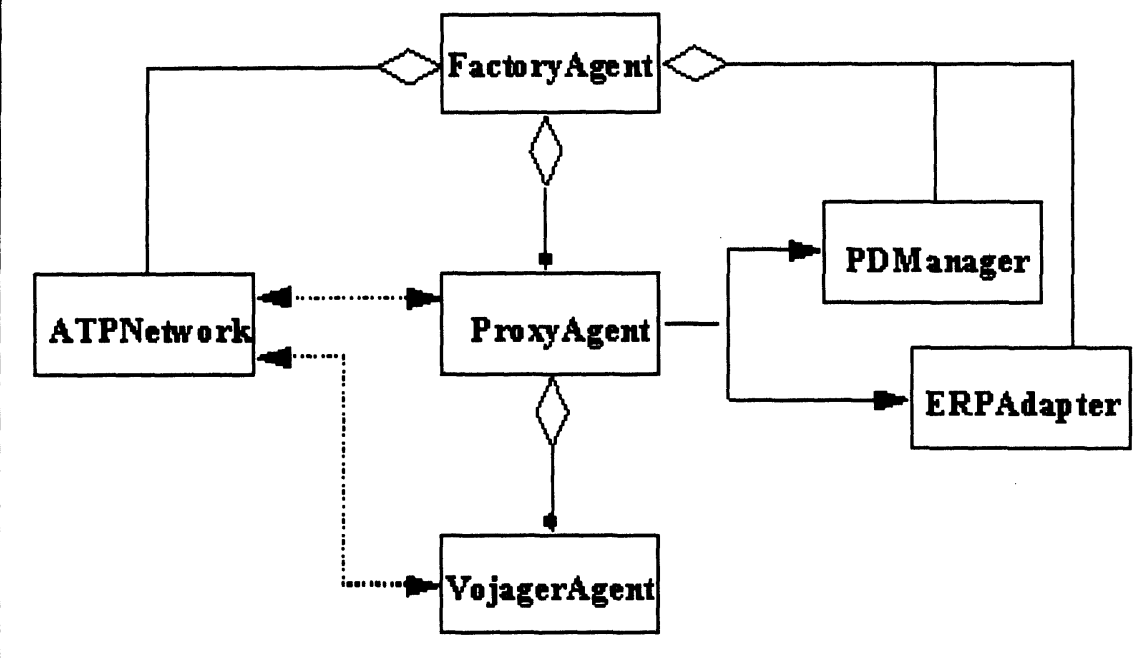

Figure 2. The design of a Factory Agent.

\subsection{Collaboration level}

The collaboration level consists of bringing together Factory Agents, each one performing a specific activity in the manufacturing/distribution process (e.g. spinning), and mobile agents (Proxy Agents and Voyager Agents) migrating between Factory Agents (see Figure 2).

\section{Factory Agents}

Factory Agents cooperate with each other to improve the overall performance of the whole system in terms of quality, costs and response time.

A typical interaction between factories is when a customer (e.g. a dyeing company) requests raw or semi-finished materials from a supplier (e.g. a weaving company). The request triggers the scheduling system of the supplier's factory (represented in Figure 2 by the class ERPADAPTER and described in Section 3.2) which has to try to register the incoming request and reply to it as quickly as possible forecasting lead times and costs for the required product. In order to process the request the supplier may have to request raw materials from another factory (e.g. a spinning company), thus triggering that company's scheduling system. When the requested item has been produced, the supplier communicates the product data to the customer. This information is retrieved from the customer database, which is represented in Figure 1 by the class PDMANAGER and will be described in Section 3.2.

FACTORYAGENT is the basic class offered by the framework to implement Factory Agents. Each Factory Agent is uniquely identified by an URL network address and provides a computational environment for the execution of mobile agents migrating through the network. In particular, the class FACTORYAGENT 
offers the support for concurrency and allows mobile agents who come from different customers to share the local Factory Agent's resources without creating visibility problems.

The framework provides the class ATPNETWORK, which hides the physical characteristics of the communication medium and provides support for the Agent Transfer Protocol (Lange and Oshima, 1998).

The class ATPNETWORK is responsible for providing the security mechanisms including authentication tools, encryption for disclosure control and integrity of data on the network, firewalls for site security, and so on. To be more precise, the security policies operate to:

- Restrict or grant agent capabilities. The affected capabilities could include creating new agents, or traveling.

- Set agent resource consumption limits. This implies CPU usage, memory and disk consumption, the number of new agents created, and the number of network connections allowed.

- Restrict or grant access. This means access to travel destinations, to operations that an agent invoke, and data that an agent can view or alter.

In a VF new customers and suppliers can dynamically appear and disappear. When a new Factory Agent enters the supply chain it establish business relationships with its suppliers by sending mobile agents (called Proxy Agent) to the suppliers Factory Agents. Each Proxy Agent acts as a proxy of the originating Factory Agent and is responsible for managing the collaboration with the supplier (e.g. informing the originating Factory Agent about changes in the supplier's schedule).

Proxy Agents

PROXYAGENT is the abstract class provided by the framework to implement Proxy Agents. The Proxy Agent constitutes the remote portion of the customer's resource planning system, and in particular it is responsible for the remote management of the events raised by the supplier Factory Agent. The Proxy Agent is able to decide which events are to be notified to the remote Factory Agent. In some cases, this architectural solution reduces the network load by buffering the events and delaying their notification. In other cases, the Proxy Agent undertakes actions to solve specific problems autonomously.

Whenever an event arises, the Proxy Agent instantiates a specific class which encapsulates the behavior in order to handle that event. The framework provides the abstract class EVENTHANDLER which has to be specialized for each event. The specific subclass of EVENTHANDLER can be specified inside the Proxy Agent before it is sent to the supplier Factory Agent, or it can be downloaded from the customer site (see Figure 3).

Proxy Agents communicate with the remote customer Factory Agent by exchanging Voyager Agents. 


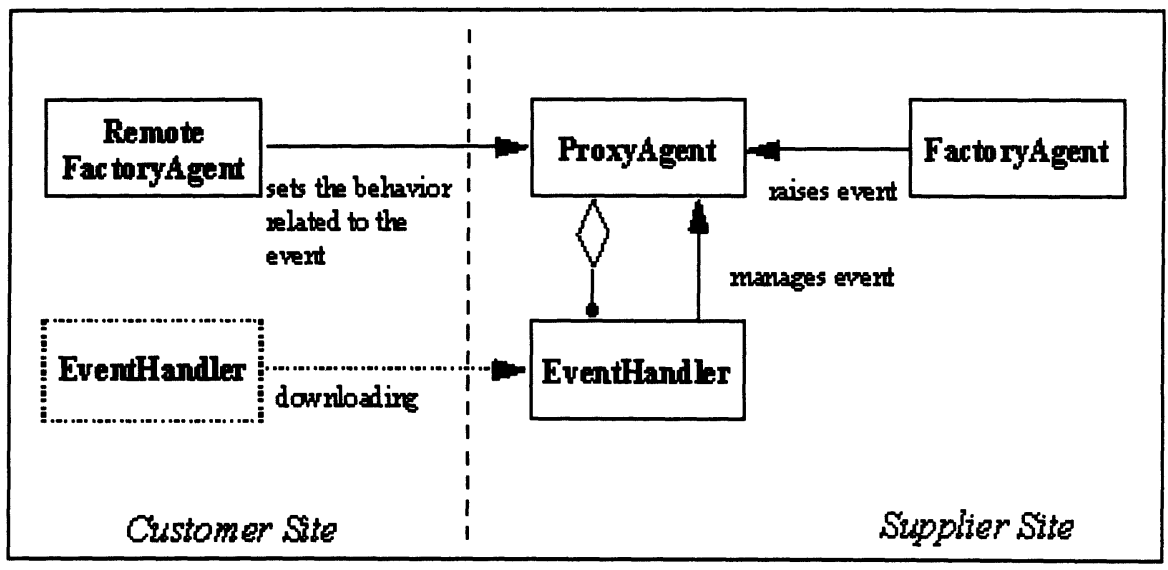

Figure 3. Event Handling by the Proxy Agent.

\section{Voyager Agent}

Voyager Agents are represented by the class VOYAGERAGENT and have three main functionalities:

- They are used to place customer orders. When the Proxy Agent receives a Voyager Agent carrying an order from the remote Factory Agent, it triggers the ERP of the local Factory Agent in order to accommodate the new request.

- They are used to send the information related to the specific material used for the production of the requested item back to the customer Factory Agent. This information is retrieved by the Proxy Agent from the Product Data Manager of the supplier Factory Agent.

- They are used to notify relevant events to the customer Factory Agent.

Performance tests have been made between two partners located in different networks. One partner was located at the Politecnico of Turin and was using a LAN connection (10 Mb/s Ethernet) for Internet access. The other partner was located in Biella (a city in the north-west of Italy) and was using a ISDN connection $(64 \mathrm{~kb} / \mathrm{s})$ for Internet Access. The time of a Voyager Agent $(4 \mathrm{~kb})$ to transport the code, post an order, and return to the customer site was about 10 seconds the first time and about 4 seconds on subsequent tests. The difference is due to the use of the caching techniques of the ATP demon: the code is transferred only the first time; on the other occasions only the state is transferred.

\subsection{Resource level}

The resource level consists of the enterprise resource planning systems and the Product Data Managers. They are specific to each physical factory and are integrated into the agent-based infrastructure by means of adapter classes.

\section{Enterprise Resource Planning system}

The resource planning systems of the enterprises belonging to the supply chain are usually heterogeneous. In order to make it possible for them to interoperate, they must have a homogeneous interface. However, legacy resource planning systems 
are in general complex, very expensive, and integrated in more general systems which manage various enterprise activities. Thus, it is not commercially feasible to substitute the enterprise's planning systems with a standard one.

For this reason the framework provides the class ERPADAPTER (see Figure 2), which has a standard interface and wraps the legacy ERP systems of the physical factories. Our work is strictly related to the ongoing business object activity inside OMG, as the ERP interface offered by the wrapper is exactly a business object.

Some of the ERPADAPTER functionalities are listed below:

- Receive a client's order;

- Receive a request to cancel a client's order;

- Receive a request to modify a client's order;

- Receive the request to speed up the execution of a client's order.

- Notify the delivery of client's order;

- Notify the anticipated delivery of (a part) order;

- Notify the delay of a client's order;

\section{Product Data Manager}

Each actor in the supply chain network up to the retailer has direct access via Internet to the situation and/or technical data of a specific item wanted (wool, yarn, fabric, and garment). Having access to an extensive database which records the seasonal production characteristics of each supplier, means that it is possible to provide regular assessments of the availability and quality of raw materials. This will contribute significantly to the customer's ability to sell his products while maintaining an acceptable level of stock.

To give an example, the key Product Data for the customer of a spinning mill are the yarn count, the composition of the yarn, the values regarding the composition of the yarn, the values thickness, thin places, naps in the yarn, values for the turn per meter in the yarn, environmental aspects of the product, and price.

Material information is stored in the local database of each enterprise, and is accessible by the Factory Agent through the PDMANAGER (see Figure 2).

\subsection{User Level}

The user level consists of the Interface Agents (see Figure 2) which provide the user with a point of access to the supply chain.

An Interface Agent is a specific mobile agent which can run inside a Web Browser. It is executed when the user loads the Web page of a specific supplier. The Interface Agents communicate with the supplier Factory Agent by exchanging Voyager Agents and offer the following services to the users:

- They allow the user to retrieve information about the characteristics of the supplier's products. This information is not statically coded in the supplier web page, but it is retrieved at run-time from the supplier's Product Data Manager. This solution has the advantage that it provides information which is always up-to-date. 
- They allow the user to forward orders for the supplier's products. In this case the interaction between the user and the supplier Factory Agent is similar to the interaction between a customer and supplier Factory Agents.

Interface Agents offer support to the development of the «virtual kiosk»; it is a point of access to the supply chain located at the retailer site. It allows customers to browse a virtual catalog (built using virtual reality techniques) of the manufacturers products and order «made-to-measure» clothes (this task is not part of our contribution to the textile project).

The adoption of virtual catalogs and the integration inside the chain of distributed PDMs offers the opportunity to transfer forecast of order data directly from the customers to weavers and spinners. One of the benefits of this architectural solution is the reduction of inventories at the retailers site.

\section{RELATED WORK}

A multi-agent framework for modeling supply chain dynamics (Swaminathan et al. 1998) has been developed at Carnegie Mellon University. It consists of a network of autonomous or semi-autonomous business entities (modeled as agents) collectively responsible for procurement, manufacturing and distribution activities associated with one or more families of related products. Different agents in the framework communicate with each other through messages. Since the framework is based on a discrete event simulator, agents are activated based on the time of activation of incoming messages. A software application using some of the concepts from this framework has been developed at IBM.

The Integrated Supply Chain Management System (Fox et al. 1994) developed at the University of Toronto provides an approach to the realtime performance of supply chain functions. The supply chain is managed by a set of interacting software agents each responsible for one or more activities in the supply chain (e.g. order acquisition, resource and activities scheduling, order dispatching, etc.) An Enterprise Information Architecture provides communication and information services.

The National Industrial Information Infrastructure Protocols (NIIIP) project (Bolton, 1996) will enable innovative suppliers throughout America's industrial base to take advantage of recent advances in object information technology (CORBA); product data definition (STEP); and communications networks (INTERNET) to assemble Virtual Enterprises. It aims to establish an open, standards based software infrastructure protocol which will integrate heterogeneous and distributed computing environments across U.S. manufacturing companies.

\section{CONCLUSION}

Collaborating autonomous agents are becoming an increasingly attractive alternative to traditional hierarchical control architectures, offering better support for robustness, scalability, reconfigurability, and reusability, especially in systems where problem solving and decision making must be distributed. This is particularly true for Virtual Factories. 
In this paper, we have shown how a supply chain integration infrastructure can be implemented using object-oriented technology, software agent technology, and code mobility.

\section{REFERENCES}

Aarsten, A., Brugali, D. and Menga, G. (1996) Designing Concurrent and Distributed Control Systems. Communication of the ACM, 39(10), 50-59.

Bell Canada Company (1996) Harvard Business Review, July/August 1996.

Bolton, R.W. (1996) Enabling the Virtual Enterprise. Technical Report National Industrial Information Infrastructure Protocols (NIIIP), http://www.niiip.org/

Brugali D. and Sycara K. (1998) Towards Agent-Oriented Application Frameworks. ACM Computing Surveys, to appear.

Deis, P. (1996) Beyond MRP with AIMS. White Paper, AIMS Software, Inc. http://www.aimserp.com/

Fox, M.S., Gruninger, M. (1994) Ontologies for Enterprise Integration. In Proc. of the 2nd Conf. on Cooperative Information Systems, Toronto, Ont., May.

Genesereth, M. and Ketchpel, S. (1994) Software Agents. Communications of the $A C M, 37(7), 48-53$.

Ghezzi, C. and Vigna, G. (1997) Mobile Code Paradigms and Technologies: A Case Study. In Proc. of the First Int. Workshop on Mobile Agents (MA'97), Berlin, Germany, April 1997. LNCS 1219, K. Rothermel and R. PopescuZeletin (Ed.), Springer-Verlag.

Lange, D.B. and Oshima, M. (1998) Programming and Deploying Mobile Agents with Java\&trade; Aglets\&trade; Addison-Wesley.

Minoura T., Pargaonkar S., and Rehfuss K. (1993) Structural active object systems for simulation. In Proc. of OOPSLA'93, Washington, D.C., October .

Nau, D.S., Herrmann, JJ.W., and Regli, W.C. (1997) Virtual Factories. In Proc of the Conference on Global Virtual Manufacturing, Detroit.

Raulefs P. (1994) The virtual factory, 13th World Computer Congress'94 Vol. 2, (ed. K. Brunnstein and E. Raubold) Elsevier Science B.V., North-Holland.

Riecken, D. (1994) Intelligent Agents. Communication of the ACM, 37(7).

Swaminathan, J.M., Smith, S.F., and Sadeh, N.M. (1998) Modeling Supply Chain Dynamics: A Multiagent Approach. Decision Sciences, forthcoming.

\section{BIOGRAPHY}

Davide Brugali is a postdoctoral research fellow at the Polytechnic of Turin, Italy, where he has received the $\mathrm{PhD}$ in Computer Science. He received the MS in Computer Science from the Polytechnic of Milan, Italy, discussing a thesis on mobile robotics. His primary research interests are innovative techniques to build and reuse software, such as Design Patterns, Application Development, Component Development, and Software Agents. His most significant publications can be found in the Communications of the ACM and ACM Computing Surveys. 
Giuseppe Menga has been professor and has held the chair of Automatic Controls at the Polytechnic of Turin since 1981. For the last ten years, his academic and professional activity has been focused on modeling and control of computerintegrated manufacturing systems, and he has pioneered the use of object-oriented technology in this field since 1985 . His most significant publications can be found in the IEEE Transactions on Automatic Control, the IEEE Transactions on Robotics and Automation, Communications of the ACM. He was the general chairman of the 1992 IEEE Robotics and Automation International Conference held in Nice (France).

Stefano Galaragga is an under-graduate student research assistant in the Dpt. of Automatica e Informatica at the Polytechnic of Turin. He is expected to graduate in computer science in 1998. 